\title{
Evolução do perfil neuromotor e capacidade funcional de mulheres fisicamente ativas de acordo com a idade cronológica
}

\author{
Sandra Mahecha Matsudo ${ }^{1}$, Victor K.R. Matsudo ${ }^{1}$, \\ Turíbio Leite de Barros Neto e Timóteo Leandro de Araújo ${ }^{1}$
}

\section{RESUMO}

Fundamentos e objetivo: Poucos estudos longitudinais têm sido feitos em mulheres fisicamente ativas para determinar o impacto do envelhecimento na aptidão física e capacidade funcional. O objetivo deste estudo foi comparar a evolução do perfil neuromotor e capacidade funcional de mulheres ativas no período de um ano, de acordo com a idade cronológica. Métodos: A amostra foi composta por 117 mulheres de 50 a 79 anos de idade ( $\overline{\mathrm{x}}$ : $65 \pm 6,6$ anos) participantes de um programa de exercícios aeróbicos, duas vezes por semana, 50 minutos por sessão durante 5,4 $\pm 3,0$ anos e divididas pela idade em: 50-59 (n: 23); 60-69 (n: 60); 70-79 (n: 34). Os testes neuromotores e de mobilidade incluíram: força muscular dos membros inferiores e superiores, agilidade, flexibilidade do tronco, velocidade de levantar-se da cadeira, equilíbrio estático, velocidade normal de andar e velocidade máxima de andar. Os resultados iniciais e nas duas avaliações seguintes, feitas a intervalos de seis meses, foram comparados usando ANOVA two way, com post-hoc Bonferroni. Resultados: Em um ano não houve nenhuma alteração no desempenho neuromotor; já a velocidade de levantar da cadeira e a velocidade de andar evidenciaram diferenças significativas nos grupos de 5059 e 60-69 anos, apresentando resultados 10-20\% melhores; quanto à velocidade máxima de andar, houve melhora (8\%) nos grupos de 60 a 79 anos. Conclusão: A evolução da aptidão física e capacidade funcional teve comportamento similar, em mulheres fisicamente ativas, indepen-

1. Projeto Longitudinal de Envelhecimento e Aptidão Física de São Caetano do Sul - Centro de Estudos do Laboratório de Aptidão Física de São Caetano do Sul - CELAFISCS - São Caetano do Sul, SP.

2. CEMAFE - Escola Paulista de Medicina - Unifesp - São Paulo, SP.

Recebido em 8/7/03

$2^{\text {a }}$ versão recebida em 1/10/03

Aceito em 3/11/03

Endereço para correspondência:

Sandra Matsudo

Av. Goiás, 1.400

09521-300 - São Caetano do Sul, SP - Brasil

E-mail: Sandra@celafiscs.com.br dentemente da idade cronológica. Essa evolução fortalece a hipótese de um efeito favorável da atividade física regular na promoção da saúde, estratégia fundamental do envelhecimento saudável.

Palavras-chave: Envelhecimento. Variáveis neuromotoras. Atividade física. Capacidade funcional. Estudo longitudinal.

\section{RESUMEN}

Evolución del perfil neuromotor y la capacidad funcional de mujeres fisicamente activas de acuerdo con la edad cronológica

Fundamentos y objetivo: Pocos estudios longitudinales han sido realizados en mujeres físicamente activas para determinar el impacto del envejecimiento de la aptitud física y la capacidad funcional. El objetivo de este estudio fué comparar la evolución del perfil neuromotor y la capacidad funcional de mujeres activas en el período de un año de acuerdo con la edad cronológica. Métodos: La muestra estuvo compuesta por 117 mujeres de 50 a 79 años de edad $(\bar{x}: 65 \pm 6,6$ años) participantes de un programa de ejercicios aeróbicos, dos veces por semana, 50 minutos por sesión durante 5,4 \pm 3,0 años y divididas por la edad: 50-59 (n: 23); 60-69 (n: 60); 70-79 (n: 34). Los tests neuromotores y de movilidad incluyeron: fuerza muscular de los miembros inferiores y superiores, agilidad, flexibilidad de tronco, velocidad de levantarse de la silla, equilíbrio estático, velocidad normal de andar y velocidad máxima de andar. Los resultados iniciales de las dos evaluaciones siguientes, realizadas intérvalos de 6 meses, fueron comparados usando ANOVA two way, con post-hoc Bonferroni. Resultados: En un ano no hubo ninguna alteración del desempeno neuromotor, ya que la velocidad de levantar de la silla y la velocidad de andar no evidenciaron diferencias significativas en los grupos de 50-59 e 60-69 años, presentando resultados un 10-20\% mejores; en cuanto a la velocidad máxima de andar hubo una mejora (8\%) en los grupos de 60 a 79 años. Conclusión: La evolución de la aptitud física y la capacidad funcional tuvo un comportamiento 
similar, en mujeres físicamente activas independientemente de la edad cronológica. Esta evolución fortalece la hipótesis de un efecto favorable de la actividad física regular en la promoción de la salud, estrategia fundamental del envejecimiento saludable.

Palabras clave: Envejecimiento. Variables neuromotoras. Actividad física. Capacidad funcional. Estudio longitudinal.

\section{INTRODUÇÃO}

Entre os 25 e 65 anos de idade há diminuição substancial da massa magra ou massa livre de gordura de 10 a $16 \%$ por conta das perdas na massa óssea, no músculo esquelético e na água corporal total que acontecem com o envelhecimento. A perda gradativa da massa do músculo esquelético e da força que ocorre com o avanço da idade, também conhecida como sarcopenia, tem sido definida por alguns autores ${ }^{1}$ como a perda de massa muscular correspondente a mais de dois desvios padrões abaixo da média da massa esperada para o sexo na idade jovem ou para outros com o mesmo critério em termos de desvio padrão, mas utilizando a massa esquelética apendicular (massa em quilogramas dividida pelo quadrado da estatura). A perda da massa muscular e, conseqüentemente, da força muscular é, ao nosso modo de ver, a principal responsável pela deterioração na mobilidade e na capacidade funcional do indivíduo que está envelhecendo. Por essa razão, tem despertado o interesse de pesquisadores a procura das causas e mecanismos envolvidos na perda da força muscular com o avanço da idade e, dessa forma, criar estratégias para minimizar esse efeito deletério e manter ou melhorar a qualidade de vida nessa etapa da vida. A sarcopenia é um termo genérico que indica a perda da massa, força e qualidade do músculo esquelético e que tem impacto significante na saúde pública pelas suas bem reconhecidas conseqüências funcionais no andar e no equilíbrio, aumentando o risco de queda e perda da independência física funcional, mas também contribui para aumentar o risco de doenças crônicas como diabetes e osteoporose. De acordo com a maioria dos estudos analisados por Cartee $^{2}$ e Porter et $_{\text {al. }}{ }^{3}$, as conclusões são bem consistentes: o tamanho da fibra do tipo II é reduzido com o incremento da idade enquanto que o tamanho da fibra do tipo I (fibra de contração lenta) permanece muito menos afetado. Na nossa opinião, tal fato se explica por serem as fibras do tipo II também muito importantes na resposta a urgências do dia-a-dia, pois contribuem com o tempo de reação e, principalmente, de resposta, que assim inviabilizariam apropriada resposta corporal para situações de emergência, como a perda súbita de equilíbrio. A perda da massa muscular é associada, evidentemente, a um decréscimo na força voluntária, com declínio de 10$15 \%$ por década, que geralmente se torna aparente somente a partir dos 50 a 60 anos de idade. Dos 70 aos 80 anos de idade tem sido relatada perda maior, que chega aos $30 \% 4$. Indivíduos sadios de 70-80 anos têm desempenho 20-40\% menor (chegando a 50\% nos mais idosos) em testes de força muscular em relação aos jovens. Essa perda do desempenho pode também ser explicada pelas mudanças nas propriedades intrínsecas das fibras musculares.

Considerando as informações expostas por Rogers e Evans ${ }^{5}$ e Booth et al. ${ }^{6}$ podemos concluir que a perda de fibras musculares, motoneurônios, unidades motoras, massa muscular e força muscular começa entre os 50-60 anos; por volta dos 80 anos idade essa perda alcançaria $50 \%$ desses componentes. Parece que os dois maiores responsáveis por esse efeito do envelhecimento são o progressivo processo neurogênico e a diminuição na carga muscular, o que poderia levar a hipotetizar que essa atrofia muscular não seria necessariamente consequiência inevitável do incremento da idade. É claro que as pessoas que se mantêm fisicamente ativas têm somente perdas moderadas da massa muscular, mas quanto dessa perda de massa muscular é conseqüência do envelhecimento e/ou a diminuição do nível de atividade física é desconhecido ${ }^{6}$.

$\mathrm{O}$ incremento e/ou a manutenção da atividade física regular pode contribuir para aumentar a expectativa de vida de mulheres idosas, mas parece ter menor benefício nas mulheres menores de 75 anos de idade 7 . Uma vez que grande parte dessas evidências epidemiológicas sustenta o efeito positivo de um estilo de vida ativo (e/ou do envolvimento dos indivíduos em programas de atividade física ou exercício) na prevenção e minimização dos efeitos deletérios do envelhecimento ${ }^{8}$, os cientistas enfatizam cada vez mais a necessidade da atividade física como parte fundamental dos programas mundiais de promoção da saúde. Não se pode pensar hoje em dia em "prevenir" ou minimizar os efeitos do envelhecimento sem que, além das medidas gerais de saúde, não se inclua também a atividade física. Essa preocupação tem sido discutida não somente nos chamados países desenvolvidos ou do Primeiro Mundo, como também nos países em desenvolvimento, como é o caso do Brasil.

No nosso Centro de Pesquisas temos desenvolvido nos últimos anos diversos protocolos de treinamento de força muscular em mulheres acima de 50 anos de idade9. Dando continuidade a esses estudos científicos, e uma vez constatada a pouca disponibilidade de dados longitudinais em países em desenvolvimento, já que alguns existem em países desenvolvidos ${ }^{10-13}$, surgiu em 1997 a idéia de iniciar um projeto longitudinal para analisar o efeito do processo de envelhecimento na aptidão física e nos níveis de atividade física e capacidade funcional. O projeto inclui a ava- 
liação de variáveis antropométricas e neuromotoras da aptidão física, avaliação da capacidade funcional, mensuração do nível de atividade física, avaliação de variáveis psicológicas (auto-imagem, perfil de estado de humor, depressão) e avaliação da ingestão alimentar.

Este estudo foi realizado com o propósito de estabelecer a evolução no período de um ano das variáveis neuromotoras da aptidão física e da capacidade funcional em mulheres fisicamente ativas de 50 a 79 anos, de acordo com a idade cronológica.

\section{METODOLOGIA}

\section{Amostra}

Os sujeitos deste estudo foram 117 mulheres de 50 a 79 anos de idade ( $\overline{\mathrm{x}}: 65 \pm 6,6$ anos) que fazem parte do Projeto Longitudinal de Envelhecimento e Aptidão Física de São Caetano do Sul coordenado pelo CELAFISCS e que desde 1997 avalia semestralmente a aptidão física e capacidade funcional de mulheres fisicamente ativas. Os sujeitos participam das atividades físicas oferecidas pelo Centro da Terceira Idade Moacyr Rodrigues da Prefeitura de São Caetano do Sul, duas vezes por semana, 50 minutos por sessão, com um tempo de prática que variou de dois a oito $\operatorname{anos}(\overline{\mathrm{x}}: 5,4 \pm 3,0$ anos) no momento da avaliação. O programa oferecido inclui exercícios aeróbicos, de alongamento e de flexibilidade e equilíbrio orientado por um profissional de educação física. A amostra deste estudo foi do tipo aleatória por conveniência e os critérios de inclusão foram: sexo feminino; maior de 50 anos de idade; aparentemente saudável; estar inscrita no programa de ginástica do Centro; estar participando de pelo menos $75 \%$ das aulas oferecidas; tempo de prática de pelo menos dois anos.

A amostra foi dividida, de acordo com a idade cronológica, assim:

Grupo de 50-59 anos: 23 mulheres

Grupo de 60-69 anos: 60 mulheres

Grupo de 70-79 anos: 34 mulheres

\section{Métodos}

Todos os testes utilizados para mensurar o perfil neuromotor da aptidão física e as variáveis de mobilidade da capacidade funcional seguiram a padronização internacional adotada pelo CELAFISCS ${ }^{14}$. Antes do inicio da pesquisa, cada uma das participantes assinou um termo de consentimento para realizar a avaliação e utilizar os dados obtidos. $\mathrm{O}$ projeto de pesquisa foi enviado e aprovado pela Comissão de Ética da Universidade Federal de São Paulo-Escola Paulista de Medicina.

As variáveis neuromotoras da aptidão física mensuradas foram: a) Força muscular dos membros superiores determinada indiretamente utilizando o teste de preensão ma- nual ou dinamometria manual com um dinamômetro marca Grip A, ajustável, com escala de 0 a 100 quilogramas; b) Força muscular dos membros inferiores medida indiretamente utilizando o teste de impulsão vertical sem auxílio dos membros superiores; c) Flexibilidade do tronco medida com o teste de sentar e alcançar utilizando um banco de madeira de 48 centímetros, com uma fita métrica de 55 centímetros fixada ao mesmo iniciando em 0 na parte mais próxima ao avaliado; d) Agilidade utilizando o teste de shuttle run; e) Equilíbrio, mensurado o equilíbrio estático com controle visual.

Os testes para medir a capacidade funcional que foram selecionados incluíram: a) Velocidade normal de andar utilizando um percurso demarcado com uma largura de 33,3 centímetros e comprimento de três metros e 33 centímetros; b) Velocidade máxima de andar em que o avaliado foi orientado a percorrer o mesmo percurso na máxima velocidade que conseguisse andar, mas sem correr; c) Velocidade de levantar-se da cadeira para medir a capacidade do indivíduo se movimentar da posição sentado para a posição em pé. A reprodutibilidade dos testes foi analisada em uma parte da amostra e os resultados evidenciaram valores de correlação de moderada a alta e significativos $(p<0,01)$ variando de 0,60 a 0,91 .

\section{Estatística}

A análise estatística para comparar a aptidão física de cada grupo etário nas três diferentes avaliações utilizou a análise de variância ANOVA two way, para mensurações repetidas dos mesmos grupos. Para localizar as diferenças foi utilizado o teste post-hoc de Bonferroni. O nível de significância adotado foi de $p<0,01$. A magnitude das possíveis diferenças entre as avaliações foi calculada com o delta percentual $(\Delta \%)$ da primeira para segunda e terceira avaliação e da segunda para a terceira avaliação. Para a análise estatística dos dados foi utilizado o software Statistical Package for the Social Sciences (SPSS) versão 10.0 for Windows.

\section{RESULTADOS}

Os resultados das variáveis neuromotoras força dos membros superiores e inferiores, flexibilidade, agilidade e equilíbrio, estão sumarizados nas tabelas 1 a 4 . Iniciando com os dados de força dos membros superiores, mensurada pelo teste de dinamometria manual (tabela 1) dos membros superiores direito e esquerdo, observamos que não foram encontradas diferenças estatisticamente significativas entre as avaliações nas três faixas etárias.

A evolução da força dos membros inferiores, determinada pelo desempenho no teste de impulsão vertical, está apresentada na tabela 2. A força muscular dos membros 
inferiores teve comportamento, durante o período avaliado, de características similares às da força dos membros superiores e da flexibilidade. Embora não fossem encontradas diferenças estatisticamente significativas entre as três avaliações nos diversos grupos etários, houve leve tendência ao incremento dos resultados ao final de um ano; assim como no caso da flexibilidade, esse aumento percentual foi diminuindo à medida que a idade cronológica avançava. Dessa forma, no grupo mais jovem (de 50-59 anos) o aumento percentual foi de quase $10 \%$, sendo de $12 \%$ no grupo de 70-79 anos. Assim, a evolução dos valores da força dos membros inferiores foi similar nos três grupos de acordo com a idade cronológica.
A flexibilidade da parte inferior do corpo apresentou tendência similar à observada com as outras variáveis neuromotoras. Conforme os dados apresentados na tabela 2, em todos os grupos etários essa variável mostrou discreto aumento, sem diferença estatística, no decorrer do ano. Em termos percentuais esse aumento foi de $15 \%$ no grupo de $50-59,6,4 \%$ no grupo de $60-69$ e de $12 \%$ no grupo de 70 79 anos.

Os resultados da agilidade corporal e do equilíbrio estático, que foram também analisados de acordo com a idade cronológica no período de um ano, estão na tabela 3.

Levando em consideração os valores da agilidade corporal (lembrando que menor tempo em segundos significa

\begin{tabular}{|c|c|c|c|c|c|c|c|c|c|c|}
\hline \multicolumn{11}{|c|}{$\begin{array}{l}\text { TABELA } 1 \\
\text { Evolução dos valores da força dos membros superiores (dinamometria) de mulheres } \\
\text { participantes do programa de exercício físico, de acordo com a idade cronológica, em } \\
\text { uma avaliação inicial (I), após um intervalo de seis meses (II) e após } 12 \text { meses (III) }\end{array}$} \\
\hline \multirow[t]{2}{*}{ Variável } & & \multicolumn{3}{|c|}{ 50-59 anos } & \multicolumn{3}{|c|}{ 60-69 anos } & \multicolumn{3}{|c|}{ 70-79 anos } \\
\hline & & $I$ & II & III & $\mathbf{I}$ & II & III & $I$ & II & III \\
\hline \multirow{4}{*}{$\begin{array}{l}\text { Dinamometria } \\
\text { direita }(\mathrm{kg})\end{array}$} & $\bar{x}$ & 26,7 & 27,9 & 28,7 & 25,2 & 25,0 & 26,8 & 25,1 & 24,1 & 25,6 \\
\hline & $\mathrm{s}$ & 6,6 & 5,2 & 4,9 & 5,2 & 4,9 & 5,3 & 5,6 & 5,6 & 5,4 \\
\hline & $\mathrm{n}$ & 22 & 23 & 23 & 60 & 59 & 60 & 34 & 34 & 34 \\
\hline & $\Delta \%$ & 4,4 & 3,1 & 7,6 & $-0,6$ & 7,1 & 6,4 & $-3,8$ & 6,0 & 2,0 \\
\hline \multirow{4}{*}{$\begin{array}{l}\text { Dinamometria } \\
\text { esquerda }(\mathrm{kg})\end{array}$} & $\bar{x}$ & 26,2 & 26,7 & 27,4 & 23,8 & 23,2 & 25,5 & 24,1 & 22,7 & 25,7 \\
\hline & $\mathrm{s}$ & 5,6 & 4,9 & 5,2 & 5,1 & 4,4 & 4,9 & 5,8 & 5,2 & 5,4 \\
\hline & $n$ & 22 & 23 & 23 & 60 & 59 & 60 & 34 & 34 & 34 \\
\hline & $\Delta \%$ & 2,0 & 2,8 & 4,9 & $-2,8$ & 10,0 & 6,9 & $-5,8$ & 13,1 & 6,6 \\
\hline
\end{tabular}

$\Delta \%$ - a ordem dos valores corresponde à variação percentual entre as avaliações: II e I-II e III-III e I.

* $\mathrm{p}<0,01$ - em relação à comparação das avaliações II e III em relação à avaliação I.

TABELA 2

Evolução dos valores da força dos membros inferiores (impulsão vertical) e da flexibilidade, de mulheres participantes do programa de exercício físicos, de acordo com a idade cronológica, em uma avaliação inicial (I), após um intervalo de seis meses (II) e após 12 meses (III)

\begin{tabular}{|c|c|c|c|c|c|c|c|c|c|c|}
\hline \multirow[t]{2}{*}{ Variável } & & \multicolumn{3}{|c|}{ 50-59 anos } & \multicolumn{3}{|c|}{ 60-69 anos } & \multicolumn{3}{|c|}{$70-79$ anos } \\
\hline & & I & II & III & I & II & III & I & II & III \\
\hline $\begin{array}{l}\text { Impulsão } \\
\text { vertical } \\
(\mathrm{cm})\end{array}$ & $\begin{array}{l}\bar{x} \\
\mathrm{~s} \\
\mathrm{n} \\
\Delta \%\end{array}$ & $\begin{array}{c}17,6 \\
3,7 \\
22 \\
9,5\end{array}$ & $\begin{array}{c}19,2 \\
5,3 \\
23 \\
0,2\end{array}$ & $\begin{array}{c}19,3 \\
5,2 \\
23 \\
9,8\end{array}$ & $\begin{array}{c}15,5 \\
3,7 \\
59 \\
3,7\end{array}$ & $\begin{array}{c}16,0 \\
4,7 \\
58 \\
2,6\end{array}$ & $\begin{array}{c}16,5 \\
3,9 \\
59 \\
6,4\end{array}$ & $\begin{array}{c}13,6 \\
3,9 \\
33 \\
-3,4\end{array}$ & $\begin{array}{c}13,2 \\
4,8 \\
33 \\
15,9\end{array}$ & $\begin{array}{c}15,3 \\
4,2 \\
33 \\
12 \\
\end{array}$ \\
\hline $\begin{array}{l}\text { Flexibilidade } \\
(\mathrm{cm})\end{array}$ & $\begin{array}{l}\overline{\mathrm{x}} \\
\mathrm{S} \\
\mathrm{n} \\
\Delta \%\end{array}$ & $\begin{array}{c}26,4 \\
9,6 \\
21 \\
9,6\end{array}$ & $\begin{array}{c}28,9 \\
9,0 \\
23 \\
-5,0\end{array}$ & $\begin{array}{l}30,4 \\
9,4 \\
23 \\
15,1\end{array}$ & $\begin{array}{c}26,5 \\
7,5 \\
59 \\
6,4\end{array}$ & $\begin{array}{c}28,2 \\
7,8 \\
59 \\
-0,6\end{array}$ & $\begin{array}{r}28,1 \\
8,4 \\
60 \\
5,8\end{array}$ & $\begin{array}{r}22,2 \\
8,7 \\
34 \\
3,2\end{array}$ & $\begin{array}{c}22,9 \\
8,2 \\
34 \\
8,9\end{array}$ & $\begin{array}{r}24,9 \\
8,5 \\
34 \\
12,3\end{array}$ \\
\hline
\end{tabular}

$\Delta \%$ - a ordem dos valores corresponde à variação percentual entre as avaliações: II e I-II e III-III e I.

* $p<0,01$ - em relação à comparação das avaliações II e III em relação à avaliação I. 
melhor desempenho), estes se mantiveram também estáveis nas avaliações nos três grupos divididos pela idade cronológica. Não foram encontradas diferenças estatisticamente significativas e a variação percentual entre a avaliação inicial e a final foi de 1,8 (no grupo mais jovem) a $2,4 \%$ (no grupo mais velho). No caso do equilíbrio estático, variável em que o melhor resultado é o valor igual a 30, os grupos de 50-59 e 60-69 anos apresentaram evolução similar à das variáveis antropométricas e à das outras variáveis neuromotoras.

A evolução do equilíbrio nesses grupos foi praticamente estável (gráfico 1), sem diferenças significativas entre as avaliações com variações percentuais nos dois grupos de $13,2 \%$ a $10 \%$. No entanto, a evolução no grupo de mulhe-

TABELA 3

Evolução dos valores da agilidade corporal e equilíbrio, de mulheres participantes do programa de exercício físico, de acordo com a idade cronológica, em uma avaliação inicial (I), após um intervalo de seis meses (II) e após 12 meses (III)

\begin{tabular}{|c|c|c|c|c|c|c|c|c|c|c|}
\hline \multirow[t]{2}{*}{ Variável } & & \multicolumn{3}{|c|}{ 50-59 anos } & \multicolumn{3}{|c|}{ 60-69 anos } & \multicolumn{3}{|c|}{ 70-79 anos } \\
\hline & & $\mathbf{I}$ & II & III & I & II & III & $\mathbf{I}$ & II & III \\
\hline \multirow{4}{*}{$\begin{array}{l}\text { Agilidade } \\
\text { (seg) }\end{array}$} & $\bar{x}$ & 17,29 & 17,38 & 17,60 & 19,12 & 19,06 & 18,89 & 21,11 & 20,75 & 21,25 \\
\hline & $\mathrm{s}$ & 1,9 & 1,4 & 1,4 & 3,0 & 3,0 & 3,1 & 3,6 & 3,0 & 4,0 \\
\hline & $\mathrm{n}$ & 22 & 23 & 23 & 59 & 60 & 59 & 33 & 34 & 33 \\
\hline & $\Delta \%$ & 0,5 & 1,3 & 1,8 & $-0,3$ & $-1,2$ & $-1,5$ & $-1,7$ & 2,4 & 0,7 \\
\hline \multirow{4}{*}{$\begin{array}{l}\text { Equilíbrio } \\
\text { (seg) }\end{array}$} & $\bar{x}$ & 20,58 & 19,59 & 21,58 & 19,55 & 19,48 & 16,98 & 10,95 & 12,12 & 9,02 \\
\hline & $\mathrm{s}$ & 11,5 & 11,1 & 9,9 & 10,5 & 9,2 & 9,5 & 9,3 & 8,0 & 7,3 \\
\hline & $\mathrm{n}$ & 20 & 23 & 23 & 58 & 59 & 59 & 29 & 33 & 34 \\
\hline & $\Delta \%$ & $-4,8$ & 10,1 & 4,9 & 0,4 & $-12,8$ & $-13,2$ & 10,7 & 25,6 & $-17,6$ \\
\hline
\end{tabular}

$\Delta \%$ - a ordem dos valores corresponde à variação percentual entre as avaliações: II e I-II e III-III e I.

* $\mathrm{p}<0,01$ - em relação à comparação das avaliações II e III em relação à avaliação inicial.

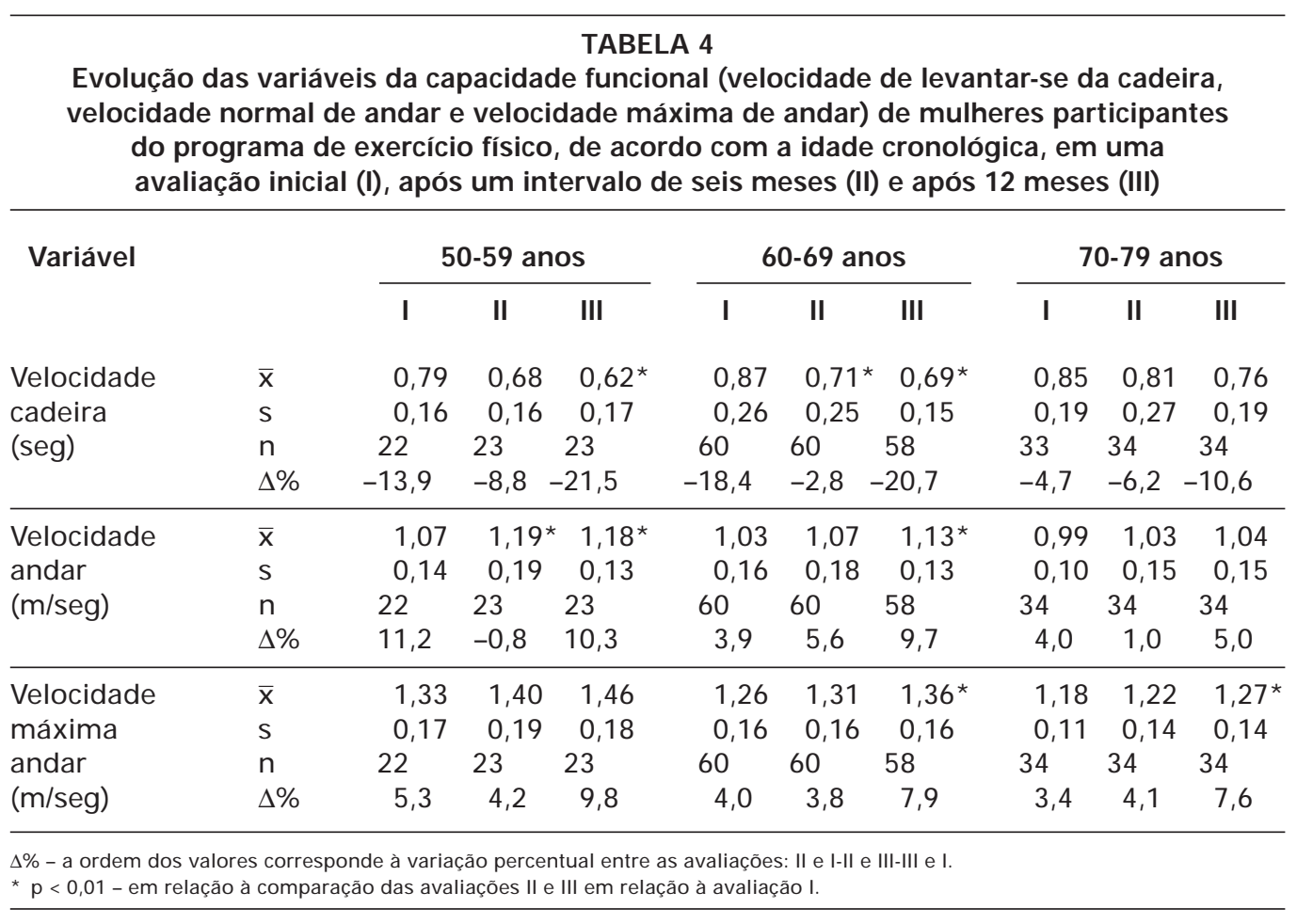


res de maior idade cronológica (70-79 anos), interessantemente, apresentou aumento do tempo, da primeira para a segunda avaliação (I x II) de praticamente $26 \%$, este não estatisticamente significativo. Entretanto, considerando a evolução da avaliação inicial à última, houve decréscimo não significativo de aproximadamente $18 \%$.

Os valores obtidos nas variáveis que mensuraram parte das funções da mobilidade geral da capacidade funcional, como a velocidade de levantar-se de uma cadeira e a velocidade de andar, estão apresentados na tabela 4.

Diferentemente da evolução verificada na maioria das variáveis antropométricas e neuromotoras, as variáveis de velocidade de levantar da cadeira e velocidade normal ao andar mostraram um padrão de evolução diferenciado de acordo com a idade cronológica. A velocidade de levantar da cadeira apresentou evolução de incremento durante o

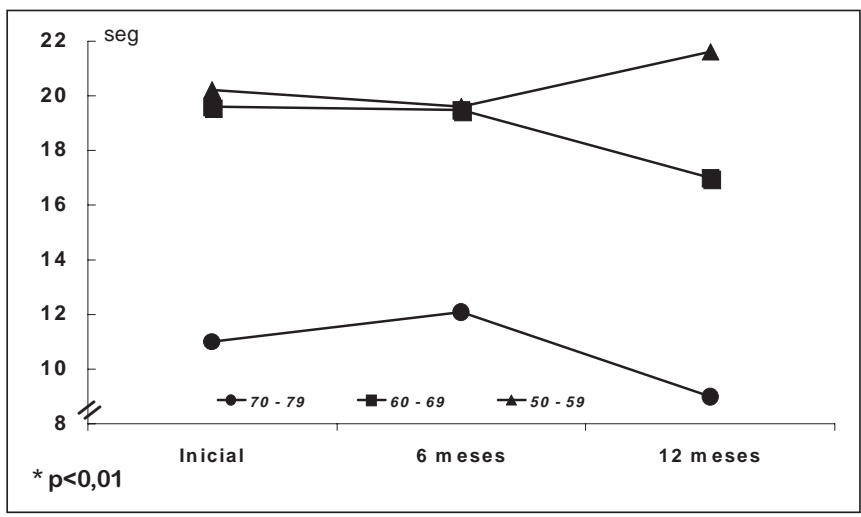

Gráfico 1 - Evolução dos valores de equilíbrio estático, de mulheres participantes do programa de exercício físico, de acordo com a idade cronológica, em uma avaliação inicial e após um intervalo de seis e 12 meses

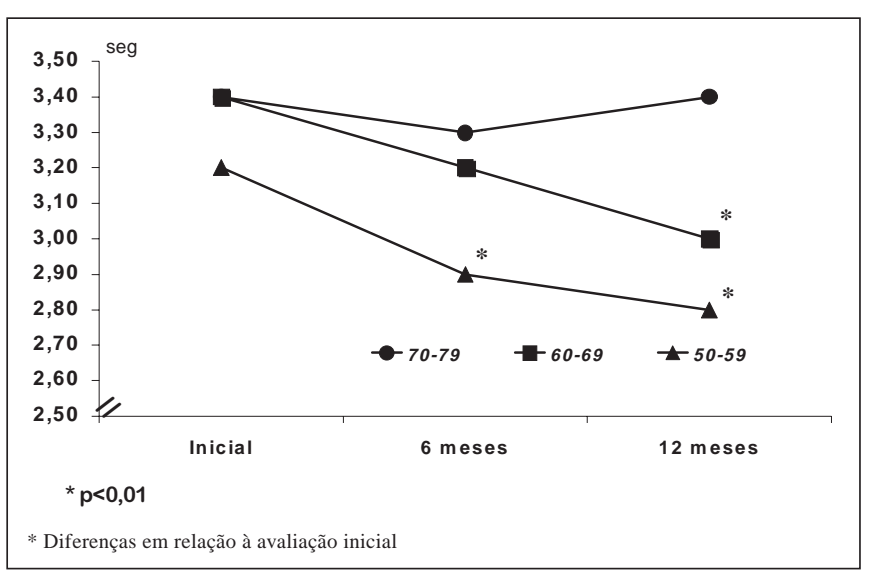

Gráfico 3 - Evolução dos valores de velocidade normal de andar, de mulheres participantes do programa de exercício físico, de acordo com a idade cronológica, em uma avaliação inicial e após um intervalo de seis e 12 meses período de avaliação de um ano, nos três grupos de idade cronológica, sendo estatisticamente significativa nos grupos de 50-59 e 60-69 anos de idade (gráfico 2).

Da primeira para a segunda avaliação, ou seja, após um intervalo de seis meses, os grupos de 50-59 anos e 60-69 diminuíram significativamente o tempo de execução do movimento em $13,5 \%$ e $18,7 \%$, respectivamente, enquanto que a diminuição percentual do tempo do grupo de 70-79 anos, não significativa, foi somente de 5,2\%. O mesmo fenômeno foi observado considerando-se a evolução no final de 12 meses, em que se verificou melhora significativa ( $p<$ 0,01) no desempenho (diminuição no tempo conseguido), que foi em torno de $21 \%$ nos grupos de 50-59 e 60-69 anos de idade.

Por outro lado, a velocidade de andar e a velocidade máxima ao andar (gráficos 3 e 4) mostraram um perfil de

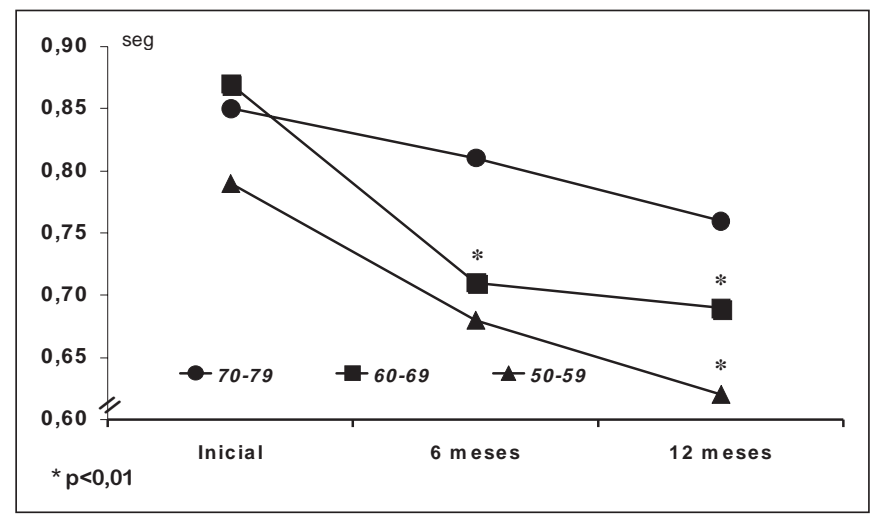

Gráfico 2 - Evolução dos valores de velocidade de levantar-se da cadeira, de mulheres participantes do programa de exercício físico, de acordo com a idade cronológica, em uma avaliação inicial e após um intervalo de seis e 12 meses

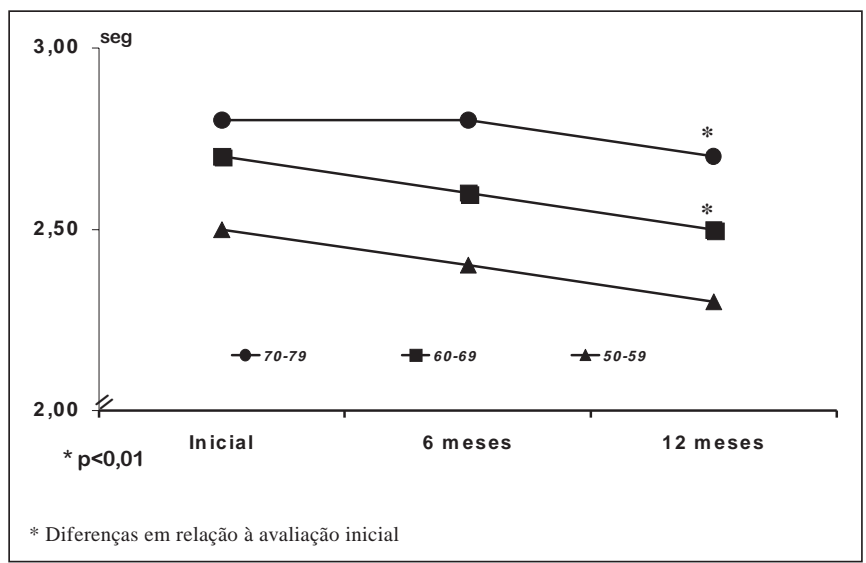

Gráfico 4 - Evolução dos valores de velocidade máxima de andar, de mulheres participantes do programa de exercício físico, de acordo com a idade cronológica, em uma avaliação inicial e após um intervalo de seis e 12 meses 
evolução diferente de acordo com a análise feita pela idade cronológica nos períodos de seis e 12 meses após a avaliação inicial.

Enquanto os resultados obtidos no teste de velocidade normal de andar apresentaram diferenças significativas nos grupos de 50-59 e 60-69 anos de idade, os valores encontrados no teste de velocidade máxima de andar, no mesmo período nos três grupos de idade cronológica, tiveram diferenças estatisticamente significativas nos grupos de 6069 e 70-79 anos de idade. Quanto aos valores de velocidade de andar na velocidade normal, observamos no grupo de 50-59 anos melhora estatisticamente significativa de aproximadamente $10 \%$ em ambas as avaliações realizadas, após seis e 12 meses da primeira avaliação. No grupo de 60-69 anos de idade a tendência foi similar, sendo verificada melhora de quase $10 \%$ da primeira para a terceira avaliação. No grupo de 70-79 anos, embora houvesse tendência de melhora de aproximadamente $4 \%$, esta não foi estatisticamente significativa.

Já os resultados conseguidos no teste de velocidade máxima ao andar nos três grupos etários evidenciaram tendência a melhorar a velocidade (em torno de 8\%); essas diferenças foram significativas estatisticamente nos grupos de 60-69 e 70-79 anos de idade.

\section{DISCUSSÃO}

As variáveis neuromotoras analisadas no estudo incluíram a força muscular dos membros superiores e inferiores, a flexibilidade do tronco, a agilidade e o equilíbrio. Considerando a evolução da força muscular, observamos que a maioria dos dados apresentados até hoje surgiu de análise de dados transversais de homens e mulheres em diferentes faixas etárias ${ }^{15,16} \mathrm{ou}$, em alguns poucos casos, de estudos feitos com intervalos de tempo de mais de uma década em amostras do sexo masculino ${ }^{17,18}$. No entanto, não encontramos na literatura disponível estudos que analisaram de forma similar a evolução dessa variável em mulheres fisicamente ativas em intervalos de seis meses ou um ano. De qualquer forma, estão bem documentados na literatura estudos com delineamentos distintos do atual e que mostram diminuição da força muscular com a idade, que é mais dramática após os 70 anos, sendo o decréscimo mais rápido nas extremidades inferiores do que nas superiores ${ }^{19}$. Monteiro et al. ${ }^{20}$, analisando um grupo de mulheres de 60 a 69 e maiores de 70 anos de idade, praticantes de atividade física, não encontraram diferenças significativas na força de preensão manual. Os valores similares de força de preensão manual entre indivíduos ativos e sedentários podem ser explicados pela participação das mãos e dos punhos nas tarefas domésticas ou outras atividades da vida diária e pela pequena ênfase no desenvolvimento da preensão manual na rotina de exercícios ${ }^{21}$. A força muscular dos membros superiores na idade adulta tem sido associada com a incapacidade funcional no período da velhice ${ }^{22}$. Os valores da força de preensão manual têm mostrado associação significativa com a incapacidade funcional: indivíduos com menores valores de força apresentaram menor velocidade de andar e risco duas vezes maior de incapacidade de autocuidado, sugerindo que a medida dessa variável na idade adulta pode servir como fator prognóstico de risco de incapacidade física na velhice. Analisando a mesma variável, Rantanen et al. ${ }^{18}$ observaram que o declínio da força muscular dos membros superiores foi quase quatro vezes maior nos indivíduos deprimidos e com peso abaixo do normal e duas vezes maior nos sujeitos deprimidos, mas com o peso normal em relação aos não deprimidos e com sobrepeso. Esses dados sugerem que a depressão e o baixo peso na terceira idade são fatores determinantes consideráveis da força muscular dos membros superiores. Se considerarmos o valor de declínio anual de 0,6 a $1 \%$ na força de preensão manual reportado na literatura ${ }^{23}$, os valores encontrados no presente estudo se mantiveram estáveis (tanto para o lado direito, quanto para o esquerdo) no período de um ano. Foram encontradas variações de até $10 \%$ que, no entanto, não foram significativas em nenhuma das três faixas etárias analisadas. Apesar de o programa de exercício físico desenvolvido no Centro da Terceira Idade não incluir atividades específicas para estimular a força de preensão manual, talvez o envolvimento regular dessas mulheres em serviços domésticos constantes e atividades da vida diária que estimulem esse tipo de força possam ter sido suficientes para manter os valores de força de preensão manual nas três faixas etárias analisadas no período de seis e 12 meses, como anteriormente descrito por Rikli e Edwards ${ }^{21}$. Da mesma forma, esses dados corroboram as observações realizadas por $\mathrm{Spirduso}^{24}$, de que a força dos músculos utilizados nas atividades diárias tendem a apresentar um padrão mais estável durante o processo de envelhecimento do que os músculos utilizados em atividades mais especializadas.

A força dos membros inferiores analisada indiretamente com o teste de impulsão vertical sem auxílio dos braços evidenciou, de forma similar ao encontrado com a força muscular dos membros superiores, um padrão de comportamento estável nos três grupos dos 50 aos 79 anos. Considerando que essa variável é fundamental para a realização das atividades cotidianas e, portanto, para a manutenção da mobilidade e da capacidade funcional durante o envelhecimento, esse padrão encontrado no grupo é extremamente positivo, haja vista que geralmente as evidências científicas apontam perda anual de $1,4 \%$ na força dos mem- 
bros inferiores ${ }^{23}$. Levando em consideração que o programa em que as senhoras estavam regularmente envolvidas não incluía exercícios com pesos para os membros inferiores, a manutenção da força muscular destes nos três grupos no período de um ano pode ser explicada pela realização de exercícios que envolviam a contração muscular dos membros inferiores, assim como pelo padrão da caminhada do grupo, além da realização de serviços domésticos que abrangiam atividades físicas vigorosas e moderadas que exercem um papel importante na manutenção da força muscular. $\mathrm{Na}$ análise realizada por Rantanen e Heikkinen ${ }^{25}$ para comparar a força muscular máxima isométrica de indivíduos de 80 anos, antes e depois de um período de cinco anos de acordo com o sexo e o nível de atividade física, ficou evidenciada diminuição significativa da massa magra e da força muscular no sexo feminino. No entanto, homens e mulheres com altos níveis de atividade física conseguiram manter a força muscular em níveis maiores que os sedentários. Nesse sentido, Lord et al. ${ }^{26}$, analisando mulheres de 57 a 75 anos de idade que se tinham envolvido em programas de exercício por períodos de mais de um ano, encontraram melhor desempenho físico entre essas mulheres, comparadas com mulheres que realizavam menos de 30 minutos de exercício por dia. Assim, esses dados também confirmam os resultados encontrados em nosso estudo referentes à manutenção da força muscular que aconteceu independentemente da idade cronológica no tempo analisado.

Dados apresentados por Andrade et al. ${ }^{16}$ com mulheres praticantes de atividade física de 30 a 73 anos de idade evidenciaram que existe decréscimo do desempenho neuromotor com o decorrer dos anos, sendo que essa perda mostrou ser maior nas variáveis de agilidade e força dos membros inferiores do que na força dos membros superiores. Considerando os dados citados pelos autores, a perda para essas variáveis dos 30 aos 73 anos foi de $67 \%$ na agilidade, $58 \%$ na força dos membros inferiores e $28 \%$ na força dos membros superiores. No entanto, as características transversais daquele estudo limitam qualquer inferência quanto aos nossos achados.

A flexibilidade do tronco, que foi analisada no presente estudo pela realização do teste de sentar e alcançar, mostrou nos três grupos de idade analisados valores que, apesar da tendência de aumento de $6 \%$ a $15 \%$, não mudaram significativamente durante o intervalo de tempo proposto. Apesar de o teste ser amplamente utilizado na área das ciências do esporte, não existe muita informação disponível da evolução dessa variável com a idade em indivíduos fisicamente ativos. Alguns dos dados disponíveis levam em consideração os efeitos no curto prazo de programas específicos de atividade física ou exercício, como o estudo similar de Petroski ${ }^{27}$, que analisou os efeitos de um programa de atividades físicas de mulheres e homens de 59 a 73 anos de idade, encontrando incremento significativo no equilíbrio estático e na flexibilidade do tronco após o período de um ano. Já com uma amostra de idosas institucionalizadas, Benedetti e Petroski ${ }^{28}$ encontraram, após um programa de exercícios de cinco meses, melhora significativa da flexibilidade, do equilíbrio e da velocidade de andar, sem alterações significativas na força de preensão manual. Outro estudo nacional que verificou os efeitos da prática da hidroginástica na aptidão física foi o realizado por Madureira e Lima $^{29}$ com mulheres de 57 a 77 anos de idade. Os autores verificaram que, após quatro meses de atividades, não houve alterações significativas na força de preensão manual e na flexibilidade do tronco. Em um estudo mais prolongado, Rikli e Edwards ${ }^{21}$ avaliaram os efeitos de três anos de um programa de exercício em mulheres previamente sedentárias de 57 a 85 anos de idade. Após um ano, os autores verificaram incremento significativo no equilíbrio estático, na flexibilidade do tronco e do ombro e na força de preensão manual, assim como melhora do tempo de reação.

Os resultados do equilíbrio estático nas diferentes faixas etárias (50-59, 60-69 e 70-79 anos) encontrados no estudo não evidenciaram diferenças estatisticamente significativas entre as avaliações realizadas, mesmo quando o grupo de mulheres de 60-69 e 70-79 anos apresentou variações positivas e negativas de $13 \%$ a $26 \%$. Esse fato pode ter ocorrido pela própria margem de erro do teste, que apresentou reprodutibilidade de $\mathrm{r}=0,75$.

No caso da amostra apresentada neste estudo, as mulheres de todas as faixas etárias conseguiram manter tanto a mobilidade quanto o nível de atividade física no período de um ano, independentemente da idade cronológica. Um efeito não esperado nas variáveis da capacidade funcional foi a melhora significativa da velocidade de andar e levantar-se da cadeira após o intervalo de 12 meses, em especial nos grupos de 50-59 e 60-69 anos. A velocidade de levantar-se da cadeira aumentou significativamente, em torno de $21 \%$ nesses dois grupos de idade, enquanto o grupo mais idoso (70-79 anos), apesar da tendência à melhora, manteve seus valores no intervalo de tempo analisado. Apresentando um padrão similar, a velocidade de andar em velocidade normal incrementou significativamente $(10 \%)$ nos mesmos grupos etários após 12 meses. Por outro lado, o comportamento da velocidade máxima de andar mostrou evolução distinta, aumentando de forma significativa em quase $8 \%$ nos grupos mais idosos (60 a 79 anos de idade). As variáveis como a velocidade de andar e de levantar-se da cadeira dependem em grande parte da força muscular dos membros inferiores, que, como afirmamos anteriormen- 
te, não foi modificada em nenhum dos grupos analisados, explicando talvez em parte por que essas variáveis da mobilidade não declinaram em nenhuma faixa etária. De acordo com os efeitos do envelhecimento na força muscular, sintetizados recentemente por Matsudo et al. ${ }^{23}$, existe declínio da força de $10-15 \%$ por década a partir dos 50 anos de idade e redução na velocidade de contração muscular que podem afetar a velocidade de andar e levantar-se da cadeira. Todavia, tal fato não aconteceu no nosso grupo no período analisado, provavelmente pela participação regular no programa de exercício e pelo nível de envolvimento com atividades domésticas.

Por outro lado, a associação entre massa muscular, força muscular e desempenho neuromotor dos membros inferiores foi analisada também por Visser et al. ${ }^{30}$. Os autores referem que entre as idades de 20 e 70 anos a massa muscular esquelética dos membros superiores e inferiores declina ao redor de $11 \%$ nas mulheres e $15 \%$ nos homens. Naquele estudo os autores mediram a massa muscular e o desempenho neuromotor dos membros inferiores utilizando o teste de velocidade de andar seis metros e a velocidade de levantar da cadeira em cinco tentativas consecutivas. Utilizando, portanto, testes similares aos empregados em nosso estudo, os autores verificaram que houve associação entre a baixa força muscular, mas não entre massa muscular, e o pior desempenho neuromotor dos membros inferiores em homens e mulheres idosas, sugerindo, dessa forma, que a força parece ser mais importante do que a massa muscular no desempenho neuromotor de atividades cotidianas, como o andar e o levantar-se da cadeira.

De acordo com revisão realizada por Daley e Spinks ${ }^{31}$, outros mecanismos que podem estar envolvidos nas alterações do equilíbrio e do andar com o envelhecimento são os limiares de sensação cutânea e proprioceptiva, que se elevam com a idade, especialmente dos membros inferiores, reduzindo a percepção de vibração da articulação do joelho. Estando no joelho boa parte dos receptores que controlam a postura, essa perda pode diminuir consideravelmente o controle do equilíbrio e age como o principal responsável pela disfunção do andar nas pessoas idosas. No entanto, de acordo com os autores, mulheres idosas que se exercitam demonstram menos desvios posturais do que as sedentárias: quanto mais ativas, menor o grau de desvio postural. No mesmo sentido, mulheres que participaram de atividades vigorosas por períodos que variaram de seis semanas a 10 anos mostraram ter melhor equilíbrio do que mulheres sedentárias da mesma idade. Esses achados ajudam a entender melhor por que foi observada em nosso estudo estabilização dos valores de equilíbrio e melhora na velocidade de andar no período de 12 meses nas mulheres de diferentes faixas etárias que estavam envolvidas re- gularmente em atividade física havia pelo menos dois anos no momento da avaliação.

De acordo com os autores anteriormente citados, a velocidade em que uma pessoa escolhe andar decresce linearmente com a idade, fenômeno que acontece não somente pela diminuição da força muscular, mas também pela diminuição na frequiência das passadas e, principalmente, pela diminuição da amplitude da passada, variáveis estas que não foram medidas no nosso estudo. Daley e Spinks ${ }^{31}$ apontam para o fato de que, após os 62 anos de idade, o declínio da velocidade de andar aumenta por década de 2,5\% e 4,5\% em homens e mulheres, respectivamente, para $16 \%$ e $12 \%$. O tamanho da passada também é significativamente alterado na terceira idade, passando de $151 \mathrm{a} 170 \mathrm{~cm}$ na população jovem para 135 a $153 \mathrm{~cm}$ nos idosos. No sexo masculino, as alterações na velocidade de andar ocorrem somente pela diminuição do comprimento da passada, enquanto as mulheres alteram tanto o tamanho como a frequiência da passada à medida que envelhecem. É um fato comum também observar incremento na dorsoflexão e diminuição da flexão plantar do tornozelo. Outros fatores relacionados positivamente com a velocidade de andar durante o envelhecimento incluem a força da panturrilha, índice de passada, horas gastas em atividades no tempo livre e altura. Já os fatores relacionados negativamente envolvem variáveis como a dor nos membros inferiores e problemas de saúde. Quando essas variáveis foram analisadas no nosso estudo, observou-se manutenção da força dos membros inferiores, do envolvimento com atividades físicas regulares e baixa prevalência de doenças, aspectos que podem fortalecer o efeito positivo encontrado na velocidade de andar. Nesse sentido, Rantanen et $a l .{ }^{32}$, analisando o equilíbrio e a força muscular como os pré-requisitos mais importantes na habilidade de andar, verificaram que o risco de incapacidade intensa de andar foi 10 vezes maior nos idosos com alterações na força muscular e no equilíbrio do que entre aqueles com alteração em somente uma dessas variáveis. Nesse cenário, as alterações no equilíbrio diminuem a participação em atividades recreacionais e serviços domésticos pelo medo de cair, o que causa, por sua vez, decréscimo na força muscular. Da mesma forma, a menor força muscular causa cansaço ou velocidade mais lenta nas pessoas que realizam esse tipo de atividades; essas dificuldades no andar e no equilíbrio fazem com que o indivíduo fique mais tempo sentado ou em repouso, fechando um ciclo vicioso e aumentando, assim, o risco de incapacidade funcional. De acordo com os dados encontrados por Frändin e Grimby ${ }^{33}$, o nível de atividade física do idoso tem correlação moderada e significativa $(0,40-0,49)$ com a velocidade máxima de andar que, por sua vez, tem alta e significativa correlação $(0,67)$ com a capacidade para subir escadas. Essas ob- 
servações corroboram plenamente as características encontradas nas senhoras pertencentes à amostra do presente estudo, já que, não tendo evidenciado diminuição na força muscular nem no equilíbrio, a habilidade de andar em um ano foi mantida ou incrementada significativamente nas diferentes faixas etárias analisadas e a realização de atividades domésticas leves e pesadas, assim como o padrão de caminhar e de subir lances de escada também foi mantido nesse intervalo de tempo.

Diversas pesquisas têm demonstrado um efeito positivo do exercício físico na velocidade de andar, como o clássico estudo de Fiatarone et al. $^{34}$, em que submeteram um grupo de idosos institucionalizados maiores de 90 anos de idade a oito semanas de treinamento de alta intensidade e encontrou-se no final incremento de $48 \%$ na velocidade de andar. Na pesquisa realizada por Spila et al. ${ }^{35} \mathrm{com}$ mulheres de 76 a 78 anos ficou evidenciado que 18 semanas de dois tipos diferentes de treinamento (de força e aeróbico) melhoraram significativamente a velocidade máxima de andar. Em outro estudo realizado com residentes de um asilo, MacRae et al..$^{36}$ determinaram o efeito de um programa de caminhada (cinco vezes por semana, 30 minutos por dia) e encontraram incremento de $77 \%$ do tempo de endurance para andar e de $92 \%$ na distância caminhada, enquanto que a velocidade de andar não sofreu nenhuma alteração significativa. No estudo de Verfaillie et al. ${ }^{37}$, comparando dois grupos de treinamento de força, com e sem treinamento específico do andar, os autores encontraram melhoras significativas na velocidade de andar, sem alteração na amplitude de passada e melhora de aproximadamente $30 \%$ na velocidade de levantar-se da cadeira, utilizando o mesmo teste de nosso estudo. Os dados sugerem que provavelmente seja necessário, além do exercício aeróbico e do treinamento de força, um programa de treinamento específico da velocidade de andar para conseguir melhoras significativas nessa variável no indivíduo que está envelhecendo, apesar de que vale lembrar que as mulheres envolvidas no presente estudo não tiveram um treinamento específico para a velocidade de andar.

No entanto, no estudo realizado por Berg e Lapp ${ }^{19}$, um programa de oito semanas de exercícios com pesos ajustáveis nos tornozelos não foi eficiente para produzir efeitos positivos na velocidade de andar, sugerindo que talvez esse tipo de treinamento de intensidade baixa a moderada, apesar de ter melhorado a força muscular, não foi suficiente para alterar a velocidade. De forma similar, Buchner et al. ${ }^{38}$ observaram que um programa de 26 semanas de treinamento aeróbico, de força e de associação de ambos, não foi suficiente para promover alterações na velocidade de andar e no equilíbrio de sujeitos de 68 a 85 anos de idade. Levando em conta essas evidências científicas, podemos pensar que exista grande variação nos resultados do efeito do exercício sobre a velocidade de andar e que, assim, não seriam surpresa os dados diferentes encontrados no nosso estudo nos três grupos de faixas etárias.

Apesar da limitada literatura nessa área, Daley e Spinks ${ }^{31}$ sugerem os mecanismos que explicam os efeitos do exercício na velocidade de andar com o envelhecimento. O exercício aeróbico e o treinamento de força têm mostrado impacto positivo nas alterações do comprimento da passada, na velocidade de andar e na média do comprimento da passada em homens idosos. No entanto, o exercício não influenciaria a velocidade de andar nos indivíduos idosos, exceto em ritmo mais lento, podendo explicar em parte os resultados encontrados em nosso estudo, que mostrou somente melhora significativa durante um ano em todas as faixas etárias na velocidade normal de andar, mas não na velocidade máxima de andar. Os autores apresentam evidências de que programas de exercício de cinco anos têm mostrado melhora na flexão e na rotação do quadril em mulheres de 50 a 71 anos, o que também explicaria parte das melhoras conseguidas com nossa amostra na velocidade de andar, já que a média de participação no programa de atividade física no momento da avaliação foi de aproximadamente cinco anos. Westhoff et al. ${ }^{39}$ encontraram que um programa de 10 semanas de treinamento de força de baixa intensidade em sujeitos maiores de 65 anos de idade foi suficiente para incrementar significativamente a capacidade funcional medida pela velocidade de levantar-se da cadeira e andar três metros, como também pela realização de algumas atividades instrumentais e não-instrumentais da vida diária, efeitos que se mantiveram pelo menos por seis meses. Mais recentemente, Puggaard ${ }^{13}$ apresentou os resultados do efeito de um programa de oito meses de exercícios na aptidão física e capacidade funcional de mulheres saudáveis divididas em grupos de 65,75 e 85 anos de idade. Os resultados evidenciaram um efeito significativo na aptidão física total, na potência aeróbica e na velocidade máxima de andar nos três grupos etários, sugerindo que os efeitos benéficos da atividade física acontecem durante o processo de envelhecimento, independente da idade cronológica, conforme apresentado nos dados do presente estudo.

Uma das explicações que deve ser levada em consideração nas alterações significativas dos dados de desempenho de algumas variáveis neuromotoras da capacidade funcional, como a velocidade de levantar-se da cadeira e a velocidade para andar, é a possibilidade do efeito de aprendizagem neural ou o efeito Hawthorne, relacionando a melhora voluntária a uma motivação psicológica ${ }^{40}$, que não foram controlados no estudo. Por outro lado, a variação dos resultados dos testes quando executados em duas ocasiões 
não pode ser descartada, já que, de acordo com a experiência de alguns autores ${ }^{35}$, o coeficiente de variação da velocidade de andar 10 metros (no presente estudo foram três metros) em duas medidas consecutivas esteve em torno de $5 \%$ e do teste de levantar-se da cadeira, em torno de $6,8 \%{ }^{37}$, valores que estão abaixo das diferenças encontradas em nosso estudo ( 8 a $21 \%$, respectivamente) após 12 meses. Todavia, é importante lembrar que a reprodutibilidade dos testes utilizados para analisar essas variáveis foi a mais baixa de toda a bateria utilizada $(r=0,60$ para a velocidade de levantar-se da cadeira e 0,73 para a velocidade de andar), fenômeno que pode de alguma forma ter interferido nos resultados finais. No estudo realizado por Stessman et $a l .{ }^{12}$ foi apresentada claramente a relação entre o nível de atividade física e o desempenho funcional nas atividades da vida diária. Os sujeitos do estudo que se exercitaram pelo menos quatro vezes por semana aos 70 anos preservaram sua capacidade funcional aos 77 , independente do estado de saúde. Em dados apresentados por Visser et al. ${ }^{10}$ em um estudo prospectivo de três anos com 2.109 homens e mulheres de 55 a 85 anos de idade, o desempenho na mobilidade (medido pelo teste de caminhar de seis metros e levantar-se da cadeira) diminui em 45,6\% da amostra. No entanto, o nível de atividade física foi associado positivamente com a mobilidade: aqueles que se mantiveram ativos tiveram menos declínio na mobilidade; os autores concluem, de forma similar à conclusão aqui apresentada, que a atividade física regular pode diminuir a velocidade de declínio do desempenho da mobilidade.

Algumas das limitações do estudo que devem ser consideradas previamente à conclusão incluem: o estudo iniciou a análise quando o grupo de mulheres já tinha começado havia pelo menos dois anos a participar regularmente no programa de exercício e não foi possível estabelecer o padrão de atividade física ao longo da vida delas, o que pode ter, sem dúvida, influenciado a evolução dessas variáveis. Da mesma forma, consideramos que, talvez, o período de um ano não seja, nesse grupo específico, um intervalo de tempo suficiente para detectar algum tipo de efeito deletério do envelhecimento, como já descrito por outros autores na população geral não fisicamente ativa. Entretanto, como o projeto, que vem sendo desenvolvido desde 1997, tem sido regularmente realizado a cada seis meses até o presente momento, estas análises poderão ser levadas a cabo no futuro. Embora não tenha sido um item efetivamente considerado dentro do delineamento do estudo, a análise simultânea de um grupo de mulheres com características socioculturais e de saúde similares às do grupo analisado, mas fisicamente inativas, poderá em análises futuras enriquecer a discussão do efeito a curto e a longo prazo da atividade física no processo de envelheci- mento. A falta de controle da vivência anterior com os testes de desempenho e a pouca experiência, no início do projeto, dos avaliadores com alguns testes de mobilidade podem ter interferido negativamente nos resultados do estudo. No entanto, em trabalhos posteriores, com maior experiência dos mesmos avaliadores, evidenciamos um padrão de reprodutibilidade dos testes similar ao encontrado em grupos de mulheres da mesma faixa etária que iniciaram programas específicos de exercício físico. Outro fator interessante que vale a pena ser considerado é que, apesar de o envolvimento com o programa de atividade física ser unicamente de duas vezes por semana (que é a disponibilidade que a prefeitura municipal tem para atender a maioria dos idosos), já existe aparentemente um efeito na manutenção da aptidão física e capacidade funcional da amostra analisada. De qualquer forma, sempre é bom lembrar que, se as evidências disponíveis na literatura apontam para uma deterioração da aptidão física e funcional com a idade, a relativa estabilidade observada nos três grupos etários nos resultados de nossa amostra no nível de atividade física ${ }^{41}$, no perfil antropométrico ${ }^{42}$ e no perfil neuromotor e de capacidade funcional (como no estudo apresentado) sugere fortemente o efeito positivo da atividade física regular, que funcionaria pelo menos como um preventivo ou redutor do declínio que acompanha o processo de senescência.

\section{CONCLUSÃO}

Dentro das características e limitações intrínsecas e extrínsecas deste estudo, nossas descobertas nos permitem concluir que: a) De forma geral, a aptidão física neuromotora, assim como a capacidade funcional, de mulheres da sexta à oitava década da vida, participantes de um programa regular de exercício físico, avaliadas a cada seis meses por um período de um ano, permaneceram estáveis; b) Melhora significativa foi observada na velocidade de andar e velocidade de levantar-se da cadeira, especialmente nos grupos de 50 a 69 anos de idade.

Os resultados sugerem que a evolução da aptidão física e capacidade funcional tem um comportamento similar, em um período de um ano, em mulheres fisicamente ativas dos 50 aos 79 anos, independentemente da idade cronológica. O conjunto desses resultados, mostrando uma evolução longitudinal estável (aptidão física) ou até positiva (capacidade funcional), fortalece a hipótese de um efeito favorável da atividade física regular como instrumento poderoso para a prevenção e promoção da saúde do idoso. O perfil encontrado sugere ainda que a participação ativa do cidadão deve provavelmente ser reavaliada, já que, como verificamos, a evolução da aptidão física e capacidade funcional de mulheres fisicamente ativas garante aparentemente, independentemente da idade cronológica, a manutenção de 
um nível funcional de independência que pode melhorar a saúde e a qualidade de vida durante o processo de envelhecimento.

Todos os autores declararam não haver qualquer potencial conflito de interesses referente a este artigo.

\section{REFERENCIAS}

1. Baumgartner R, Koehler K, Gallagher D, Romero L, Heymsfield S, Ross $\mathrm{R}$, et al. Epidemiology of sarcopenia among the elderly in New Mexico. Am J Epidemiol 1998; 147:755-63.

2. Cartee GD. Influence of age on skeletal muscle glucose transport and glycogen metabolism. Med Sci Sports Exerc 1994;26:577-85.

3. Porter M, Vandervoort A, Lexell J. Aging of human muscle: structure, function and adaptability. Scand J Med Sci Sports 1995;5:129-42.

4. Fiatarone-Singh MA. Body composition and weight control in older adults. In: Lamb DR, Murray R, editors. Perspectives in exercise science and sports medicine: exercise, nutrition and weight control. Carmel: Cooper, 1998;11:243-88.

5. Rogers M, Evans W. Changes in skeletal muscle with aging: effects of exercise training. Exerc Sport Sci Rev 1993;21:65-102.

6. Booth F, Weeden S, Tseng B. Effect of aging on human skeletal muscle and motor function. Med Sci Sports Exerc 1994;26:556-60.

7. Greeg EW, Cauley JA, Stone K, Thompson TJ, Bauer DC, Cummings SR, et al. Relationship of changes in physical activity and mortality among older women. JAMA 2003;289:2379-86.

8. American College of Sports Medicine. Position stand on exercise and physical activity for older adults. Med Sci Sports Exerc 1998;30:992-1008.

9. Raso V, Andrade E, Matsudo S, Matsudo V. Exercício com pesos para mulheres idosas. Rev Bras Ativ Fis Saúde 1997;2:17-26.

10. Visser M, Pluijm SM, Stel VS, Bosscher RJ, Deeg DH. Physical activity as a determinant of change in mobility performance: the longitudinal aging study Amsterdam. J Am Geriatr Soc 2002;50:1774-81.

11. Marcell TJ, Hawkins S, Tarpenning KM, Hyslop DM, Wiswell RA. Longitudinal analysis of lactate threshold in male and female master athletes. Med Sci Sports Exerc 2003;355:810-7.

12. Stessman J, Hammerman-Rozenberg R, Maaravi Y, Cohen A. Effect of exercise on ease in performance activities of daily living and instrumental activities of daily living from age 70 to 77 : the Jerusalem longitudinal study. J Am Geriatr Soc 2002;50:1934-8.

13. Puggaard L. Effects of training on functional performance in 65,75 and 85 year-old women: experiences deriving from community based studies in Odense, Denmark. Scand J Med Sci Sports 2003;13:70-6.

14. Matsudo S. Avaliação do idoso - Física e funcional. Londrina: Midiograf, 2000.

15. Dâmaso A, Figueira Jr A, Ferreira M. Terceira idade: força de preensão manual em senhoras na faixa etária entre 50-79 anos. In: Anais da II Bienal de Ciências do Esporte. São Caetano do Sul, Brasil: CELAFISCS, 1991;34.

16. Andrade E, Matsudo S, Matsudo V. Performance neuromotora em mulheres ativas. Rev Bras Ativ Fis Saúde 1995;1:5-14.

17. Frontera W, Hughes V, Fielding R, Fiatarone M, Evans W, Roubenoff R. Aging of skeletal muscle: a 12-yr longitudinal study. J Appl Physiol 2000; 88:1321-6.

18. Rantanen T, Penninx B, Masaki K, Lintunen T, Foley D, Guralnik JM. Depressed mood and body mass index as predictors of muscle strength decline in old men. J Am Geriatr Soc 2000;48:613-7.

19. Berg W, Lapp B. The effect of a practical resistance training intervention on mobility in independent, community-dwelling older adults. J Aging Phys Act 1998;6:18-35
20. Monteiro W, Amorim P, Farjalla R, Farinatti P. Força muscular e características morfológicas de mulheres idosas praticantes de um programa de atividades físicas. Rev Bras Ativ Fis Saúde 1999;1:20-8.

21. Rikli R, Edwards D. Effects of a three-year exercise program on motor function and cognitive processing speed in older women. Res Q Exerc Sport 1991;62:61-7.

22. Rantanen T, Guralnik JM, Foley D, Masaki K, Leveille S, Curb JD, et al. Midlife hand grip strength as a predictor of old age disability. JAMA 1999;281:558-60.

23. Matsudo S, Matsudo V, Barros T. Impacto do envelhecimento nas variáveis antropométricas, neuromotoras e metabólicas da aptidão física. Rev Bras Ciênc e Mov 2000;8:21-32.

24. Spirduso W. Physical dimensions of aging. $1^{\text {st }}$ ed. Champaign: Human Kinetics, 1995.

25. Rantanen T, Heikkinen E. The role of habitual physical activity in preserving muscle strength from age 80 to 85 years. J Aging Phys Act 1998; 6:121-32.

26. Lord S, Caplan G, Ward J. Balance, reaction time, and muscle strength in exercising and nonexercising older women: a pilot study. Arch Phys Med Rehabil 1993;74:837-9.

27. Petroski EL. Efeitos de um programa de atividades físicas na terceira idade. Rev Bras Ativ Fis Saúde 1997;3:34-40.

28. Benedetti T, Petroski E. Idosos asilados e a prática de atividade física. Rev Bras Ativ Fis Saúde 1999;3:5-16.

29. Madureira A, Lima S. Influência do treinamento físico no meio aquático para mulheres na terceira idade. Rev Bras Ativ Fis Saúde 1999;3:59-66.

30. Visser M, Deeg D, Lips P, Harris T, Bouter L. Skeletal muscle mass and muscle strength in relation to lower-extremity performance in older men and women. J Am Geriatr Soc 2000;48:381-6.

31. Daley M, Spinks W. Exercise, mobility and aging. Sports Med 2000;29: 1-12.

32. Rantanen T, Guralnik JM, Ferruci L, Leveille S, Fried LP. Coimpairments: strength and balance as predictors of severe walking disability. J Gerontol A Biol Sci Med Sci 1999;54:M172-6.

33. Frändin K, Grimby G. Assessment of physical activity, fitness and performance in 76-year-olds. Scand J Med Sci Sports 1994;4:41-6.

34. Fiatarone M, Marks E, Ryan N. High-intensity strength training in nonagenarians: effects on skeletal muscle. JAMA 1990;263:3029-34.

35. Spila S, Multanen J, Kallinen M, Era P, Suominen H. Effects of strength and endurance training on isometric muscle strength and walking speed in elderly women. Acta Physiol Scand 1996;156:457-64.

36. MacRae P, Asplund L, Schnelle J, Ouslander J, Abrahamse A, Morris C. A walking program for nursing home residents: effects on walk endurance, physical activity, mobility, and quality of life. J Am Geriatr Soc 1996;44:175-80

37. Verfaillie D, Nichols J, Turkel E, Hovell M. Effects of resistance, balance, and gait training on reduction of risk factors leading to falls in elders. J Aging Phys Act 1997;5:213-28.

38. Buchner D, Cress M, De Lateur B, Esselman P, Margherita A, Price R, et al. The effect of strength and endurance training on gait, balance, fall risk, and health services use in community-living older adults. J Gerontol A Biol Sci Med Sci 1997;52:218-24.

39. Westhoff M, Stemmerik L, Boshuizen H. Effects of a low-intensity strength-training program on knee-extensor strength and functional ability of frail older people. J Aging Phys Act 2000;8:325-42.

40. Morganti C, Nelson M, Fiatarone M, Dallal G, Economos C, Crawford $\mathrm{B}$, et al. Strength improvements with $1 \mathrm{yr}$ of progressive resistance training in older women. Med Sci Sports Exerc 1995;27:906-12.

41. Matsudo S, Matsudo V, Araújo T. Perfil do nível de atividade física e capacidade funcional de mulheres maiores de 50 anos de idade de acordo com a idade cronológica. Rev Bras Ativ Física Saúde 2001;6:12-24.

42. Matsudo S, Matsudo V, Barros Neto T. Perfil antropométrico de mulheres maiores de 50 anos fisicamente ativas de acordo com a idade cronológica - evolução de um ano. Rev Bras Ciênc e Mov 2002;10:21-32. 